\title{
Lysosome and Cytoskeleton Pathways Are Robustly Enriched in the Blood of Septic Patients: A Meta-Analysis of Transcriptomic Data
}

\author{
Jie Ma, ${ }^{1,2}$ Chuanxi Chen, ${ }^{1,2}$ Andreas S. Barth, ${ }^{3}$ Chris Cheadle, ${ }^{1}$ \\ Xiangdong Guan, ${ }^{2}$ and Li Gao ${ }^{1}$ \\ ${ }^{1}$ Division of Allergy \& Clinical Immunology, Johns Hopkins University School of Medicine, Baltimore, MD 21224, USA \\ ${ }^{2}$ Department of Critical Care Medicine, The First Affiliated Hospital of Sun Yat-Sen University, China \\ ${ }^{3}$ Division of Cardiology, Johns Hopkins University School of Medicine, Baltimore, MD 21287, USA \\ Correspondence should be addressed to Xiangdong Guan; carlg@163.net and Li Gao; lgao2@jhmi.edu
}

Received 19 December 2014; Revised 27 March 2015; Accepted 1 April 2015

Academic Editor: Tânia Silvia Fröde

Copyright (C) 2015 Jie Ma et al. This is an open access article distributed under the Creative Commons Attribution License, which permits unrestricted use, distribution, and reproduction in any medium, provided the original work is properly cited.

\begin{abstract}
Background. Sepsis is a leading cause of mortality in intensive care units worldwide. A better understanding of the blood systems response to sepsis should expedite the identification of biomarkers for early diagnosis and therapeutic interventions. Methods. We analyzed microarray studies whose data is available from the GEO repository and which were performed on the whole blood of septic patients and normal controls. Results. We identified 6 cohorts consisting of 450 individuals ( $\operatorname{sepsis}=323$, control $=$ 127) providing genome-wide messenger RNA (mRNA) expression data. Through meta-analysis we found the "Lysosome" and "Cytoskeleton" pathways were upregulated in human sepsis patients relative to controls, in addition to previously known signaling pathways (including MAPK, TLR). The key regulatory genes in the "Lysosome" pathway include lysosomal acid hydrolases (e.g., protease cathepsin A, D) as well as the major (LAMP1, 2) and minor (SORT1, LAPTM4B) membrane proteins. In contrast, pathways related to "Ribosome", "Spliceosome" and "Cell adhesion molecules" were found to be downregulated, along with known pathways for immune dysfunction. Overall, our study revealed distinct mRNA activation profiles and protein-protein interaction networks in blood of human sepsis. Conclusions. Our findings suggest that aberrant mRNA expression in the lysosome and cytoskeleton pathways may play a pivotal role in the molecular pathobiology of human sepsis.
\end{abstract}

\section{Introduction}

Sepsis, a maladaptive response to infection, is a common and lethal syndrome. The hospital incidence of sepsis has been reported as high as $153 / 100,000$ to $353 / 100,000$ in both industrialized [1,2] and also developing countries [3], with an increasing trend year by year [1-3]. In the United States, sepsis is one of the top ten leading causes of mortality [4]. Although adjusted in-hospital mortality has decreased gradually (2-3\% per year) according to a recent report $[1,5]$, sepsis associated mortality remained high, from 50/100,000 to $75 / 100,000$ [1, 6]. It was even higher when sepsis was accompanied by organ dysfunction, ranging from $23 \%$ to $58 \%$ with dysfunction of one organ $[2,3,7]$ and increasing to $77.4 \%$ when three or more organs had failure [3]. Moreover, the respiratory system tended to be the most commonly infected site (49.3\%) [1] and was associated with a high rate of organ failure (47.6-91.2\%) $[2,8]$. Elucidating the early pathogenesis of sepsis will likely decrease time to diagnosis and intervention, which would be expected to improve outcomes in critically ill patients.

The systemic inflammatory response syndrome (SIRS) contributes to multiple organ failure (multiple organ dysfunction syndrome) in sepsis and is accompanied by a first pro-inflammatory response and a following "immune paralysis" (compensatory anti-inflammatory response syndrome) responsible for secondary infections. This widespread and excessive reaction induces microthrombi formation, capillary obstruction, microcirculatory alterations, tissue edema by capillary leak, and neutrophil recruitment leading to multiple tissue damages, organ failures and finally to death [9]. 
There are three recognized stages of severe host response to pathogen with progressively increased mortality rates: sepsis, severe sepsis, and septic shock. Known pathways related to innate immune effectors, inflammatory mediators, and modulators of coagulation are particularly implicated in sepsis pathophysiology $[10,11]$. The toll-like receptor (TLR) signaling pathway is not only relevant to immunosuppression but also to increasing neutrophil migration, vascular permeability, and proinflammatory cytokines in sepsis [12, 13]. The MAPK and NF- $\kappa \mathrm{B}$ signaling cascades, induced by the inflammatory mediators lipopolysaccharide (LPS) and TNFalpha in models of lung inflammation and sepsis, are known to promote endothelial cell hyperpermeability [14, 15]. In addition, inhibition of the NF- $\kappa \mathrm{B}$ signaling pathway in combination with antibiotics improved the survival rate in a sepsis model of cecal ligation and puncture (CLP) in rats, likely by reducing inflammation and attenuating vascular endothelial leakage [16-18]. Thus, activation of the procoagulant, proinflammatory, and proapoptotic pathways at the blood and tissue interface is one of the most prominent features of sepsis $[19,20]$.

Gene expression profiles enable systems-wide analysis of cellular processes and have provided novel insights on the molecular mechanisms underlying sepsis as will be discussed below. Genome-wide transcription profiling of human sepsis in host leukocytes revealed a consistent theme of activation of pathogen recognition pathways (including pathogen recognition receptors TLRs and CD14) and signal transduction pathways, which are processes essential for subsequent transcription of immune response genes [11]. In order to better understand the pathogenesis of sepsis at the transcriptional level and with the aim of translating emerging genomics data into new early diagnosis and effective treatment strategies, we used bioinformatics approaches to integrate the results from multiple microarray studies of sepsis. We selected datasets, which are freely available in the GEO (Gene Expression Omnibus, http://www.ncbi.nlm.nih.gov/geo/) data repository [22]. We chose datasets based on peripheral whole blood samples from patients with sepsis and from controls since blood is readily accessible and likely to reflect changes in disease related pathways.

\section{Materials and Methods}

2.1. Study Selection and Data Mining. We searched GEO for experiments studying sepsis in human whole blood samples and identified 6 studies (GEO accession numbers: GSE30119, GSE8121, GSE9692, GSE28750, GSE13015 and GSE21802; Table 1(a)) fulfilling the following criteria: (1) sample size in each study should have $n \geq 5$ in both the groups of sepsis patients and controls; (2) had whole genome-coverage (>20,000 genes) in each study; and (3) had low variance across arrays. All 6 studies were previously published [2328 ] and were included in the meta-analysis (Figure 1). The 6 cohorts met the above selection criteria had included a total of 450 individuals (patients with sepsis $=323$, controls $=127$ ) providing genome-wide mRNA expression data (Table 1(a)).
Patient characteristics, source of infection and time of data collecting of included studies were summarized in Table 1(b).

2.2. Identification of Common Pathways for Human Sepsis. All 6 studies were analyzed separately using the original Series Matrix File data format provided in GEO. Information on processing of samples including source of total RNA isolation, microarray hybridization protocol and data normalization methods were presented in Table S1 in Supplementary Material available online at http://dx.doi.org/10.1155/2015/ 984825. First, we used unpaired two-class Significance Analysis of Microarray (SAM) [29] to identify differentially expressed transcripts within each study (using log2normalized microarray data and permutations $=200$ ), which corresponds to ANOVA with correction for multiple testing. Differences in gene expression were regarded as statistically significant if a false discovery rate (FDR) of $q<0.1$ was achieved, as previously described [30]. Second, we used the Kyoto Encyclopedia of Genes and Genomes (KEGG) (http:// www.genome.jp/kegg/kegg2.html) [31] for pathway mapping. Briefly, results for each dataset from the SAM analysis were submitted to DAVID (http://david.abcc.ncifcrf.gov/) [32] under the functional annotation option specifying Homo sapiens as the species. Additionally, the threshold was modified as count $=1$ and EASE-score $=10$ to find every possible KEGG pathway [33]. Net expression of a pathway, defined as the number of upregulated transcripts minus downregulated transcripts expressed as percentage of the total number of genes within a KEGG pathway, was calculated to enable comparison of KEGG pathways representing a different number of genes, as described previously [33]. Third, rank scores were given to the top 10 up or downregulated KEGG pathways in each study (with rank scores assigned from 1 to 10,10 represented the most significant pathway) and a top KEGG pathway was defined if it had the highest combined rank scores and most appearances across all studies. Finally, we visualized the net expression using Genesis software [34].

\subsection{Identification of Candidate Genes in Top Common Path-} ways for Human Sepsis. We further analyzed each GEO dataset separately to identify individual differentially expressed genes. First, we extracted 122 genes in the lysosome and 215 genes in the cytoskeleton pathways in KEGG from the normalized dataset. Second, we estimated the mean difference between the average expressions of those genes per sample and used Student's $t$-test to determine statistically significant differential expression (both up- and downregulation) between sepsis patients and the controls, and this was done for all genes in both pathways for each dataset. Then we calculated the average $P$ values for both pathways in each dataset (reported as $-\log (P)$ in Figure 3). Lastly, we reported significantly upregulated genes in sepsis patients satisfying a criteria of a $t$-test $P$ value $<0.01$ and a fold change (FC) $>1.5$ (Table 3 ).

2.4. Network Analysis of Protein-Protein Interactions. Analysis of protein-protein interactions were conducted using the "Unified Human Interactome Database" (UniHI 7) [21], which contains more than 350,000 molecular interactions between genes, proteins, and drugs, as well as numerous 
TABLE 1: (a) GEO series included in the study for sepsis in human. (b) Patient characteristics, source of infection, and time of data collection.

(a)

\begin{tabular}{llcc}
\hline $\begin{array}{l}\text { GEO } \\
\text { accession }\end{array}$ & Comparison groups & Probe ID & Publication (PMID) \\
\hline GSE30119 & Healthy $(n=44)$ versus sepsis $(n=99)$ & 48802 & 22496797 \\
\hline GSE8121 & $\begin{array}{l}\text { Nonseptic }(n=15) \text { versus septic shock_day } 1(n=30) \text { versus septic } \\
\text { shock_day } 3(n=30)\end{array}$ & 54675 & 17932561 \\
\hline GSE9692 & Normal $(n=15)$ versus septic shock $(n=30)$ & 54675 & 18460642 \\
\hline GSE28750 & Healthy $(n=20)$ versus Sepsis $(n=10)$ versus post-surgical $(n=11)$ & 54675 & 21682927 \\
\hline & $\begin{array}{l}\text { Platform6106: nonsepsis }(n=19) \text { versus septicemic melioidosis }(n=24) \\
\text { versus sepsis_other infection }(n=24) \\
\text { GSE13015* }\end{array}$ & $\begin{array}{l}\text { Platform6947: nonsepsis }(n=10) \text { versus melioidosis }(n=16) \text { versus } \\
\text { sepsis_other infection }(n=13)\end{array}$ & 48687 \\
\hline GSE21802 & $\begin{array}{l}\text { Healthy }(n=4) \text { versus sepsis without MV }(n=16) \text { versus sepsis with } \\
\text { MV }(n=20)\end{array}$ & 48803 \\
\hline
\end{tabular}

(b)

\begin{tabular}{llll}
\hline $\begin{array}{l}\text { GEO } \\
\text { accession }\end{array}$ & Patients characteristics & Source of infection & Time of data collection \\
\hline & & $\begin{array}{l}\text { Blood and marrow or arthritis } \\
(n=56)\end{array}$ & $\begin{array}{l}\text { Different days during } \\
\text { hospitalization }\end{array}$ \\
GSE30119 & Age $>10$ and $<18$ year-old & Lung $(n=11)$ & $\leq 3$ days $(n=34)$ \\
& Septic pediatrics & Skin $(n=10)$ & $3-7$ days $(n=43)$ \\
& Only 77 patients had identified & $\geq 7$ days $(n=22)$ \\
\end{tabular}
positive infecting organism

$\begin{array}{ll}\text { Blood (15) } & \\ \text { Lung (3) } & \text { (1) Within } 24 \mathrm{~h} \text { of admission to ICU } \\ \text { Urine (1) } & \text { (day 1) } \\ \text { Retropharyngeal abscess (1) } & \text { (2) } 48 \mathrm{~h} \text { after day } 1 \text { (day 3) } \\ \begin{array}{l}\text { Only 20 patients had identified } \\ \text { positive infecting organism }\end{array} & \end{array}$

$\begin{array}{ll}\text { GSE8121 } & \text { Age }<10 \text { year-old } \\ \text { Septic shock pediatrics }\end{array}$

\section{Blood (10)}

Lung (1)

$\begin{array}{ll}\text { GSE9692 } & \text { Age }<10 \text { year-old } \\ \text { Septic shock pediatrics }\end{array}$

Cerebral spinal fluid (1)

Other (2)

Retropharyngeal abscess (1)

Within $24 \mathrm{~h}$ of admission to ICU

Only 15 patients had identified

positive infecting organism

\begin{tabular}{|c|c|c|c|}
\hline GSE28750 & $\begin{array}{l}\text { Age }>18 \text { year-old } \\
\text { BMI }<40 \\
\text { Septic patients }\end{array}$ & All blood & $\begin{array}{l}\text { Septic patients: within } 24 \mathrm{~h} \text { of } \\
\text { admission } \\
\text { Post-surgical patients: within } 24 \mathrm{~h} \\
\text { following surgery }\end{array}$ \\
\hline GSE13015* & $\begin{array}{l}\text { Age }>18 \text { year-old } \\
\text { Septic patients }\end{array}$ & All blood & Within 7 days of diagnose \\
\hline GSE21802 & $\begin{array}{l}\text { Age }>18 \text { and }<65 \text { year-old } \\
\text { Septic patients with confirmed } \\
\text { H1N1 infection }\end{array}$ & Lung $(n=19)$ & $\begin{array}{l}\text { Early period (before day } 9 \text { in the } \\
\text { course of the disease) } \\
\text { Late period (from day } 9 \text { in the } \\
\text { course of the disease) }\end{array}$ \\
\hline
\end{tabular}

$\bar{*}$ GSE13015 was a published study using 2 different microarray platforms and in different patients, therefore was considered as two separate studies in the statistical analysis.

other types of data such as gene expression and functional annotation. UniHI 7 constitutes one of the most comprehensive platforms for the query, visualization, and analysis of molecular networks in human. UniHI7 also allows the filtering of protein-protein interactions and provides "Phenotype
Enrichment analysis," thus enabling the construction of phenotype-specific networks. The display (Figure 5) was restricted to at least 3 literature references in PubMed. We also explored whether known drug targets and the phenotype of "Mortality and Aging" were enriched with network proteins. 


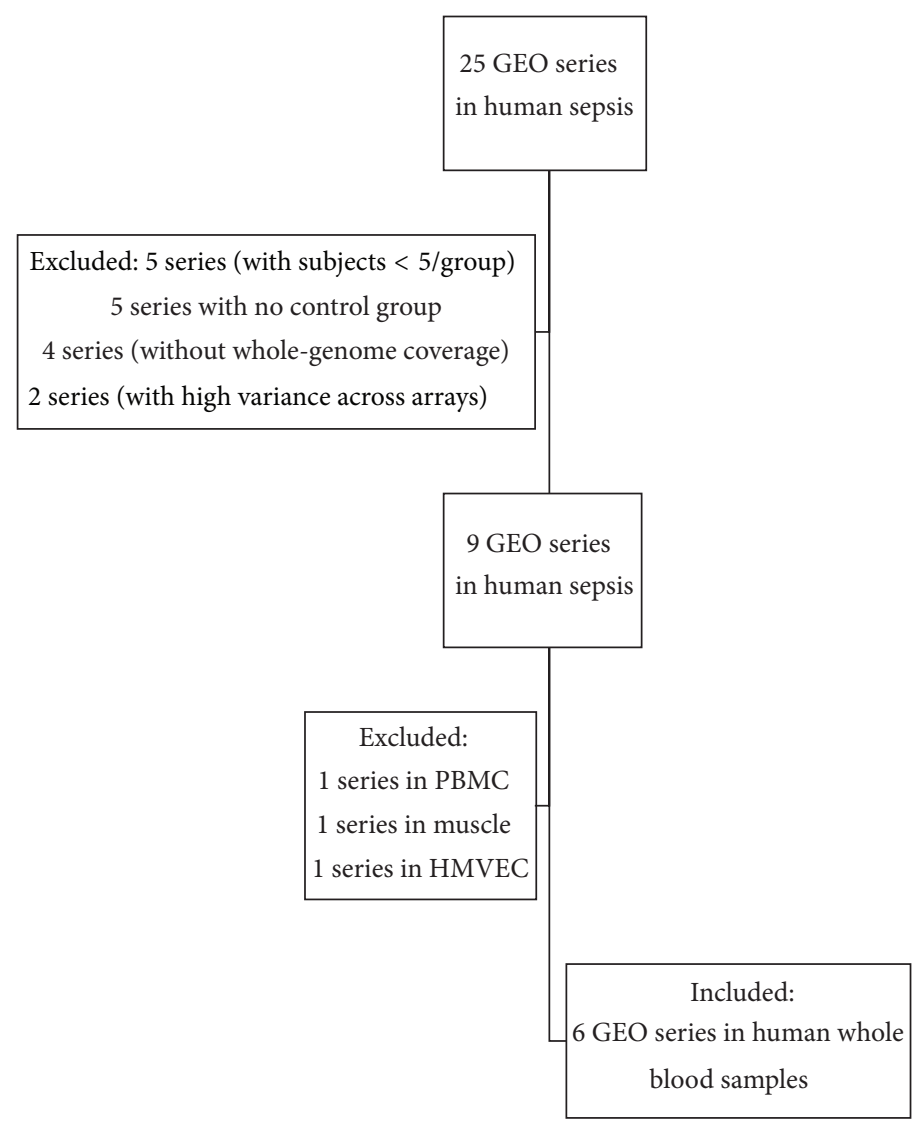

FIGURE 1: Selection of existing GEO series. A total of 25 GEO series in human were obtained using the key word "sepsis" and only 9 series met the criteria. We included 6 out of 9 series, which used whole blood samples, 3 series which used other tissue types (PBMC, muscle, and HMVEC) were excluded.

\section{Results}

3.1. Pathways Enriched in Individual Studies. We first summarize the results found in the studies we examined and then describe the results of our meta-analysis in relation to these findings. The predominant pathogen was bacteria in 5 out of 6 published studies while one study (GSE21802) focused on influenza virus (with confirmed H1N1 infection). The primary sources of infection were blood and lung. Shanley et al. [23] found time-dependent, differential regulation of genes involved in multiple signaling pathways and gene networks primarily related to immunity and inflammation in pediatric patients with septic shock $(n=30)$, relative to controls $(n=15)$. Notably, interleukin-6, toll-like receptor, p38 MAP kinase, and NF- $\kappa \mathrm{B}$ signaling pathways were upregulated, on both day 1 and day 3, while antigen presentation, natural killer cell, and T cell receptor signaling pathways were downregulated. These findings were subsequently confirmed in an additional cohort of 30 children with septic shock and 14 separate normal controls [24]. Other studies aimed at detecting genes for early diagnosis of sepsis also demonstrated that genes directly involved in innate and early adaptive immune function (56\%), cell cycling and white blood cell differentiation (32\%), and extracellular matrix remodeling (7\%), as well as immune modulation (5\%), may serve as molecular biomarkers [25]. Another study of septicemic melioidosis, a severe infectious disease caused by the Gram-negative bacillus Burkholderia pseudomallei, identified a signature significantly enriched in genes coding for products involved in MHC class II antigen processing and presentation pathways [26]. The largest study was on children hospitalized with community-acquired $S$. aureus infection $(n=99)$ and an overall transcriptional signature of overexpression of innate immunity and hematopoiesis related genes as well as underexpression of genes related to adaptive immunity was identified [28]. In summary, there were critically important pathways representing an imbalance of pro- and anti-inflammation, immune dysfunction and modulation across the aforementioned studies.

3.2. Common Pathways Revealed in Septic Patients through Meta-Analysis. Out of a total of 429 KEGG pathways in the KEGG database, we identified 199 KEGG pathways with statistically significant regulation across the 6 studies in human sepsis we examined (listed in Supplementary Figure 1). The 199 KEGG pathways with statistically significant regulation displayed more consistent transcriptional patterns among the 6 studies with bacterial infection as compared to study GSE21802 that had virus infection. Sepsis is defined as systemic inflammatory response syndrome with infection 
TABLE 2: Whole blood transcriptional profiles identified top up- and downregulated pathways in human sepsis.

\begin{tabular}{|c|c|c|c|}
\hline Upregulated pathways & Rank scores* & Downregulated pathways & Rank scores* \\
\hline Lysosome & 47 & Ribosome & 69 \\
\hline Regulation of actin cytoskeleton & 46 & Spliceosome & 55 \\
\hline Pathways in cancer & 45 & Antigen processing and presentation & 35 \\
\hline MAPK signaling pathway & 34 & Cell adhesion molecules & 34 \\
\hline Fc gamma R-mediated phagocytosis & 26 & Intestinal immune network for IgA production & 31 \\
\hline Chemokine signaling pathway & 23 & Graft-versus-host disease & 28 \\
\hline Toll-like receptor signaling & 17 & Purine metabolism & 21 \\
\hline Neurotrophin signaling pathway & 16 & Autoimmune thyroid disease & 15 \\
\hline Insulin signaling pathway & 16 & Allograft rejection & 14 \\
\hline Focal adhesion & 14 & $\mathrm{~T}$ cell receptor signaling pathway & 13 \\
\hline Leukocyte transendothelial migration & 12 & Cytokine-cytokine receptor interaction & 9 \\
\hline Alzheimer's disease & 12 & Primary immunodeficiency & 8 \\
\hline Endocytosis & 8 & Natural killer cell mediated cytotoxicity & 8 \\
\hline Olfactory transduction & 8 & Viral myocarditis & 7 \\
\hline Cell cycle & 7 & Hematopoietic cell lineage & 7 \\
\hline Renal cell carcinoma & 6 & RIG-I-like receptor signaling pathway & 7 \\
\hline Oocyte meiosis & 6 & RNA degradation & 6 \\
\hline Chronic myeloid leukemia & 5 & Jak-STAT signaling pathway & 6 \\
\hline Complement and coagulation cascades & 5 & Type I diabetes melitus & 4 \\
\hline \multirow[t]{3}{*}{ Oxidative phosphorylation } & 4 & Phosohatidylinositol signaling system & 4 \\
\hline & & Fc epsilon RI signaling pathway & 2 \\
\hline & & Inositol phosphate metabolism & 1 \\
\hline
\end{tabular}

* Pathways were listed according to rank scores (from high to low). Rank scores were given to the top 10 up- or downregulated KEGG pathways in each study (with rank scores assigned from 1 to 10,10 represented the most significant pathway) and a top KEGG pathway was defined if it had the highest combined rank scores and most appearances across all studies.

and the pathogen includes bacteria, fugal, and virus. The heterogeneity we observed in the host response signatures for GSE21802 is likely influenced by the type of pathogen (e.g., virus versus bacteria). According to rank scores and frequency of appearance, we found robust upregulation of multiple KEGG pathways in whole blood samples of human sepsis across all studies examined, including "Lysosome," "Regulation of actin cytoskeleton," "Pathways in cancer," "MAPKinase signaling," "Fc gamma R-mediated phagocytosis," "Chemokine signaling pathway," "Toll-like receptor signaling," "Neurotrophin signaling pathway," "Insulin signaling pathway," and "Focal adhesion" (Figure 2 and Table 2). In contrast, the top 4 downregulated pathways included "Ribosome," "Spliceosome," "Antigen processing and presentation," and "cell adhesion molecules" (Table 2). Of note, in addition to well-known pathways involved in sepsis (e.g., MAPK signaling and Toll-like receptor signaling), our study identified novel signaling pathways "Lysosome" and "Regulation of actin cytoskeleton" as the two most significantly upregulated pathways across all 6 studies, suggesting that these 2 pathways might play a previously underrecognized role in human sepsis. More importantly, our study revealed an imbalance between protein synthesis (e.g., downregulation of "Ribosome" and "Spliceosome" pathways) and lysosomemediated protein degradation (e.g., upregulation of "Lysosome" pathway), which may account for the multiple organ injury and failure in sepsis.
We recognize that the gene expression signature we identified through meta-analysis could be tissue- and disease stage-specific. We had restricted our selection criteria to include only studies performed on whole blood of human sepsis; this approach could reduce the heterogeneity in host response signatures due to various tissue types. However, the time of sample/data collection for each study varied, which could influence the precise patterns of temporal gene expression. This remained as one of the limitations of our study arising from the challenge of finding sufficient number of studies available in GEO for stratifying patients into more homogeneous populations.

\subsection{Candidate Genes Differentially Expressed in Lysosome and} Cytoskeleton Pathways. Given that lysosome and cytoskeleton were novel pathways for sepsis and among the most significantly upregulated in our study, we further examined individual genes dysregulated in these 2 pathways, the relation/interaction among them and their utilities as potential biomarkers for sepsis. We identified significantly upregulated genes in both the lysosome (a) and cytoskeleton (b) pathways across multiple studies by comparing the mean of average expression of each gene in the group of sepsis patients relative to the controls. Both the $-\log P$ values and fold changes are displayed for the top 15 genes most significantly upregulated across studies (Table 3). Interestingly, genes encoding multiple lysosomal acid hydrolases including serine protease 


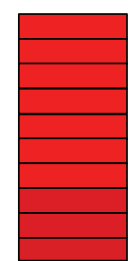

hsa03810:Regulation of actin cytoskeleton

hsa04142:Lysosome

hsa04010:MAPK signaling pathway

hsa04910:Insuling signaling pathway

hsa04666:Fc gamma R-mediated phagocytosis

hsa04510:Focal ashesion

hsa04144:Endocytosis

hsa04062:Chemokine signaling pathway

hsa04620:Toll-like receptor signaling pathway

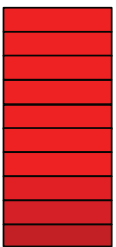

hsa05200:Pathways in cancer

hsa04010:MAPK signaling pathway

hsa04810:Regulation of actin cytoskeleton

hsa04062:Chemokine signaling pathway

hsa04142:Lysosome

hsa05220:Chronic myeloid leukemia

hsa04722: Neurotrophin signaling pathway

hsa04910:Insuling signaling pathway

hsa04510:Focal ashesion

hsa04144:Endocytosis

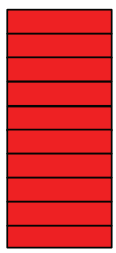

hsa04666:Fc gamma R-mediated phagocytosis hsa 04722: Neurotrophin signaling pathway sa05200:Pathways in cance

hsa04010:MAPK signaling pathway

hsa04910:Insuling signaling pathway

hsa04142:Lysosome

hsa04620:Toll-like receptor signaling pathway

hsa04210:Apoptosis

hsa04810:Regulation of actin cytoskeleton

hsa05212:Pancreatic cancer

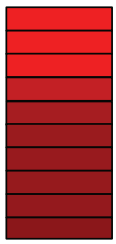

hsa03810:Regulation of actin cytoskeleton

hsa04510:Focal ashesion

hsa 05200:Pathways in cancer

hsa 05010:Alzheimer's disease

hsa05010:Alzheimer's disease

hsa00190: Oxidative phosphorylation

hsa04142:Lysosome

hsa04144:Endocytosis

hsa03320:PPAR signaling pathway

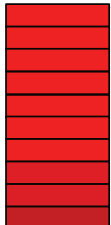

hsa05200:Pathways in cance

hsa04062:Chemokine signaling pathway

hsa03810:Regulation of actin cytoskeleton

hsa04142: Lysosome

hsa04666:Fc gamma R-mediated phagocytosis

hsa04010:MAPK signaling pathway

hsa04670:Leukocyte tranendothelial migration

hsa05215:Prostate cancer

hsa04810:Regulation of actin cytoskeleton

hsa05200:Pathways in cancer

hsa04142:Lysosome

hsa04670:Leukocyte tranendothelial migration

hsa04010:MAPK signaling pathway

hsa 04666:Fc gamma R-mediated phagocytosis

hsa04620:Toll-like receptor signaling pathway

hsa04062:Chemokine signaling pathway

hsa04722:Neurotrophin signaling pathway
hsa04510:Focal ashesion

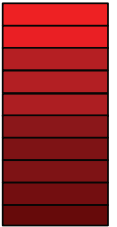

hsa 04810:Regulation of actin cytoskeleton hsa04142:Lysosome

hsa04740:Olfactory transduction

hsa04110:Cell cycle

hsa04114: Oocyte meiosis

hsa05010:Alzheimer's disease

hsa00010:Alzheimer's disea

hsa00010:Glycolysis/Gluconeogenesis

hsa04360: Axon guidance

hsa05130:Pathogenic Escherichia coli infection
hsa04020:Calcium signaling pathway
GSE30119

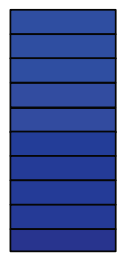

hsa03010:Ribosome

hsa03040:Spliceosome

hsa00230:Purine metabolism

hsa05332:Graft-versus-host disease

hsa04612:Antigen processing and presentation

hsa04672:Intestinal immune network for IgA production

hsa03018:RNA degradation

hsa05320:Autoimmune thyroid disease

hsa05330:Allograft rejection

hsa05340:Primary immunodeficiency

GSE8121

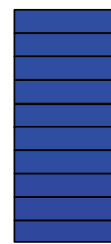

hsa03040:Spliceosome

hsa03010:Ribosome

hsa04612:Antigen processing and presentation

hsa 04514:Cell adhesion molecules (CAMs)

hsa 05332:Graft-versus-host disease

hsa04672:Intestinal immune network for IgA production

hsa05320:Autoimmune thyroid disease

hsa05320:Autoimmune thyro

hsa05330:Allograft rejection

hsa04940:Type I diabetes mellitus

GSE9692

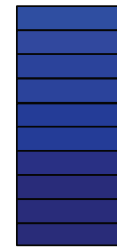

hsa03010:Ribosome

hsa03040:Spliceosome

hsa04612:Antigen processing and presentation

hsa 05416:Viral myocarditis

hsa 04514:Cell adhesion molecules (CAMs)

hsa04672:Intestinal immune network for IgA production

hsa 04640:Hematopoietic cell lineage

hsa05320:Autoimmune thyroid disease

hsa05340:Primary immunodeficiency

hsa00230:Purine metabolism

GSE28750

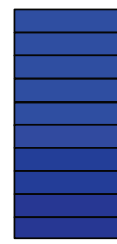

hsa03010:Ribosome

hsa03040:Spliceosome

hsa04660:T cell receptor signaling pathway

hsa00230: Purine metabolism

hsa04612:Antigen processing and presentation

hsa04672:Intestinal immune network for IgA production

hsa 05340:Primary immunodeficiency

hsa05330:Allograft rejection

hsa05332:Graft-versus-host disease

hsa03010:Ribosome

hsa03040:Spliceosom

hsa04514:Cell adhesion molecules (CAMs)

hsa04672:Intestinal immune network for IgA production

hsa05330:Allograft rejection

hsa05332:Graft-versus-host disease

hsa05320:Autoimmune thyroid disease

hsa04612:Antigen processing and presentation

hsa04940:Type I diabetes mellitus

hsa00230:Purine metabolism

GSE13015-

GPL6947

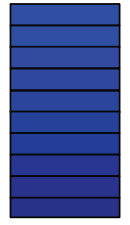

hsa03010:Ribosome

hsa03040:Spliceosome

hsa05332: Graft-yersus-host disease

isa04514:Cell adhesion molecules (CAMs)

hsa04612:Antigen processing and presentation

hsa04672: Intestinal immune network for IgA production

hsa00230:Purine metabolism

hsa05330:Allograft rejection

hsa03018:RNA degradation

hsa04940:Type I diabetes mellitus

GSE21802

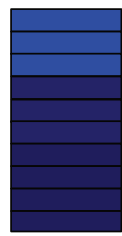

hsa03010:Ribosome

hsa 04060:Cytokine-cytokine receptor interaction

hsa04650: Natural killer cell mediated cytotoxicity

hsa 04622:RIG-I-like receptor signaling pathway

hsa04630:Jak-STAT signaling pathway

hsa 04660:T cell receptor signaling pathway

hsa04070:Phosphatidylinositol signaling system

hsa04070:Phosphatidylinositol signa

hsa04664:Fc epsilon RI signaling pathway

hsa00562:Inositol phosphate metabolism

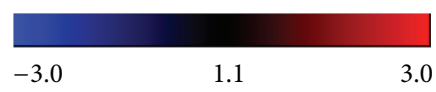

FIGURE 2: Top 10 up- and downregulated KEGG pathways in human sepsis. KEGG pathways were sorted ascending for upregulation and descending for downregulation by net expression and visualized in Genesis software. Here we only showed top ten pathways that had either the highest or the lowest net expression in each individual study. Blue color represents negative net expression and red color means positive net expression. The absolute net expression value is proportionate with color brightness, the brighter the color the higher the absolute value.

cathepsin A (CTSA) and aspartic proteases cathepsin D (CTSD), galactosidase, alpha (GLA), and sulfatase (GNS) as well as the major (LAMP1 and LAMP2) and minor (SORT1 and LAPTM4B) lysosomal membrane proteins are among the top dysregulated molecules. Additionally, vacuolar ATPase
(ATP6AP1), which is responsible for proton transport, was also activated leading to acidification, which then activates hydrolytic enzymes [35]. Overall, these findings suggested that the lysosomal activities were increased in human sepsis (Figures 3 and 4). According to our search of the PubMatrix 


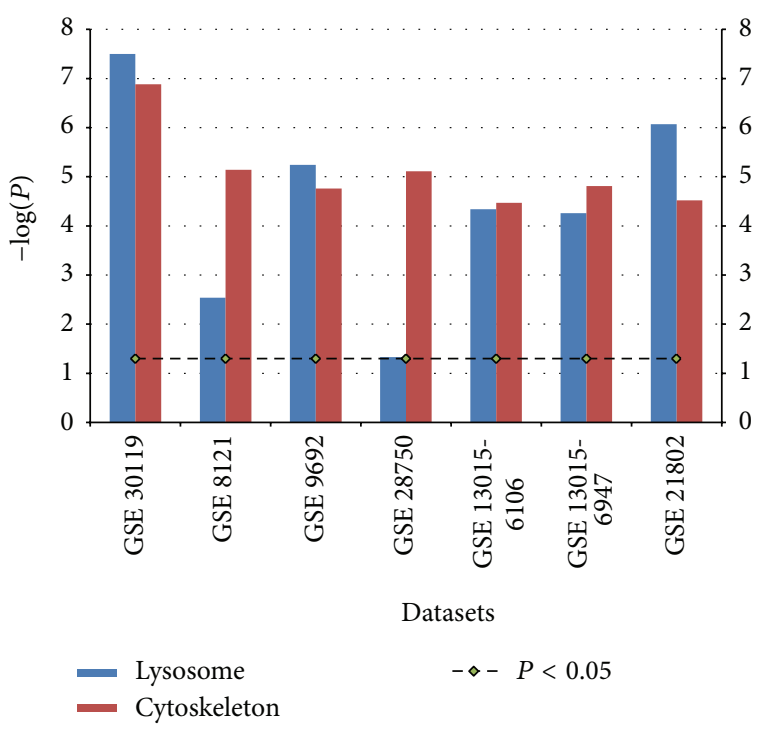

FIGURE 3: Lysosome and cytoskeleton pathways are enriched across multiple studies in blood of septic patients. This figure displays the $-\log P$ values (with $P<0.05$ as cut-off) yielded from Student's $t$ test for the mean of average expression of genes in each pathway, comparing sepsis patients to the controls in each dataset, and only significantly up-regulated genes with either $P$ value $<0.01$ or FDR $>1.5$ were included in the analysis.

database (http://pubmatrix.grc.nia.nih.gov/), 9 out of the 15 upregulated genes in the lysosome pathway (including GLA, CD63, GNS, and LAMP2) and 7 in the cytoskeleton pathway (including CD14, integrins, and MAPK1) have been reported in the literature associated with search terms "sepsis," "severe sepsis," or "septic shock" (Table 3 ).

As a proof of concept, we further evaluated whether these significantly differentially expressed transcripts or their coding protein products previously had been utilized as biomarkers for sepsis. Currently the measurement of plasma C-reactive protein (CRP) and procalcitonin (PCT) has been utilized in diagnosing and monitoring neonatal sepsis, severe sepsis and septic shock, at the onset and in the course of the disease [36]. Commercial methods for the automated measurement of the soluble CD14 subtype presepsin (sCD14ST) and lipopolysaccharide binding protein (LBP), the two old biomarkers, have been proposed over the past years to manage critically ill newborns with acute inflammation and sepsis. Presepsin levels in blood could also predict bacteremia in patients with SIRS in the Emergency Department [37]. Neutrophil activation marker CD63 was upregulated in patients with sepsis, suggesting that circulating neutrophils are fully activated in sepsis [38]. Another study which evaluated leukocyte activation in sepsis showed that cell surface expression of all activation markers (CD11b, ICAM-1, CD66b, CD63, and CD64) was increased on both neutrophils and monocytes from sepsis patients compared to healthy controls [39]. However, many genes in these two pathways have remained largely unexplored, and our results provide evidence for their involvement in the transcriptomic changes in human sepsis. We further evaluated all upregulated genes

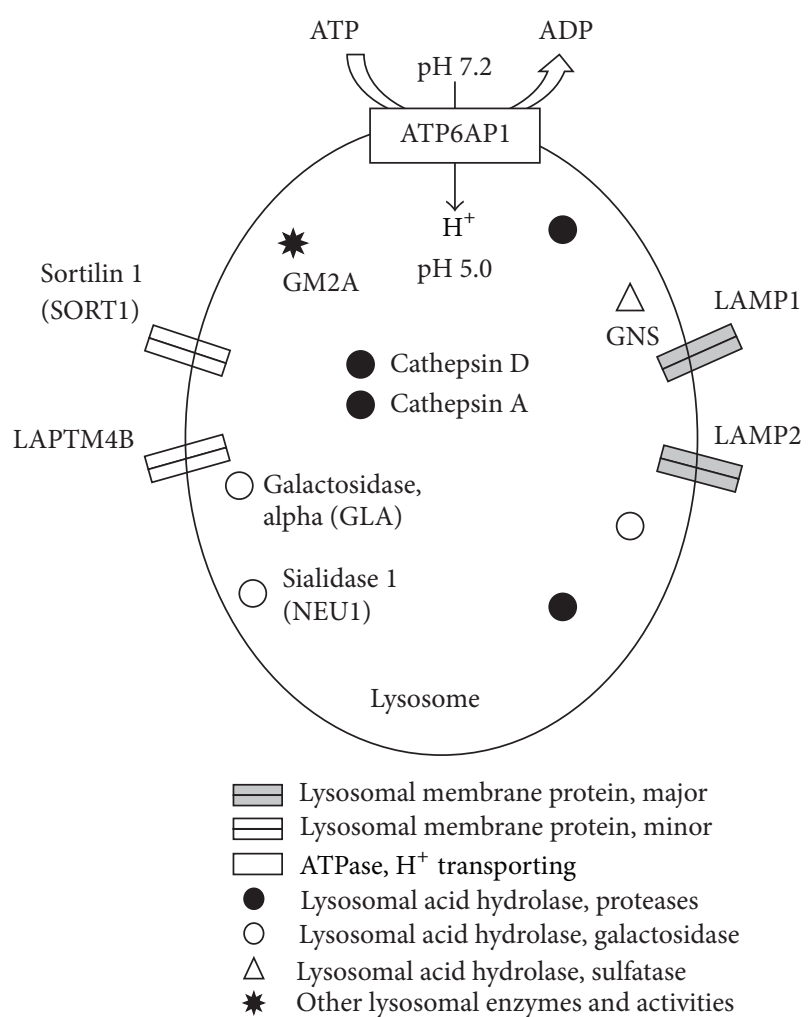

Figure 4: Target molecules in lysosome pathway dysregulated in human sepsis. Lysosome is a cytoplasmic organelle containing enzymes that break down biological polymers. Organization of the lysosome is depicted as dense spherical vacuole containing a variety of acid hydrolases (close circle: proteases; open circle: galactosidase; open triangle: sulfatase) that are active at the acidic $\mathrm{pH}$ maintained within the lysosome. Furthermore, both the major (double rectangle boxes in grey) and minor (double rectangle boxes) lysosomal membrane proteins are involved. ATPase $\left(\mathrm{H}^{+}\right.$ transporting) is displayed as open rectangle and "other lysosomal enzymes and activities" are displayed as close star.

in these two pathways in each study, comparing sepsis patients to the controls in each dataset, and found that the lysosome and cytoskeleton pathways are overall dysregulated across all studies in blood of septic patients (Figure 3).

\subsection{Network Analysis for Enrichment of Mortality Phenotype} and Drug Targets. The network-based approaches can provide the most unbiased analysis of high-throughput omics data (e.g., gene expression array), a better understanding of human diseases on the systems-level [40], and help networkrelated drug design. Among different molecular networks, protein-protein interaction (PPI) networks have emerged as an important resource for understanding data from gene expression or proteomics experiments. Three consecutive steps are typically required to perform PPI network analysis [40]. The first step is to identify genes (e.g., differentially expressed genes) or proteins of interest. In the second step, these inputs (also known as "seed proteins") are used to search and retrieve binary interactions from a curated PPI database. The third step is network analysis such as the topology 
TABLE 3: Top 15 upregulated genes in lysosome (a) and cytoskeleton (b) pathways across seven datasets. Genes demonstrated a fold-change of greater than 1.5 and $P$ value of less than 0.05 (the $-\log P$ values are shown in parentheses) are displayed.

(a) Lysosome pathway

\begin{tabular}{|c|c|c|c|c|c|c|c|c|c|}
\hline $\begin{array}{l}\text { Gene } \\
\text { Symbol }\end{array}$ & Gene Name & $\# 1$ & $\# 2$ & $\# 3$ & $\# 4$ & $\# 5$ & $\# 6$ & $\# 7$ & PubMatrix $^{*}$ \\
\hline SORT1 & Sortilin 1 & $2.16(13)$ & $4.05(9.8)$ & $4.48(10.5)$ & $5.19(8.4)$ & $4.86(4.4)$ & $7.82(2.3)$ & - & 0 \\
\hline CTSD & Cathepsin D & $1.86(10.9)$ & $2.57(7)$ & $2.36(6)$ & $2.1(8)$ & $2.75(8.8)$ & - & $5.94(5.9)$ & 3 \\
\hline SLC11A1 & $\begin{array}{l}\text { Solute carrier family } 11 \\
\text { (proton-coupled } \\
\text { divalent metal ion } \\
\text { transporter), member } 1\end{array}$ & $3.69(5.6)$ & $2.62(6.5)$ & $3.98(8.4)$ & $2.4(5)$ & $3.31(5.3)$ & $3.85(4.7)$ & - & 4 \\
\hline CD63 & CD63 molecule & $2.02(5.9)$ & $3.35(8.4)$ & $3(10.5)$ & $2.84(8.7)$ & $2.82(6.1)$ & - & - & 38 \\
\hline GNS & $\begin{array}{l}\text { Glucosamine } \\
\text { (N-acetyl)-6-sulfatase }\end{array}$ & - & $3.17(5.9)$ & $3.91(8.3)$ & $2.76(6.3)$ & $1.85(5.8)$ & - & $2.77(4.4)$ & 10 \\
\hline GM2A & $\begin{array}{l}\text { GM2 ganglioside } \\
\text { activator }\end{array}$ & - & $1.85(4.2)$ & $2.55(6.5)$ & $2.42(7.6)$ & $3.11(5)$ & $6.17(3.7)$ & - & 0 \\
\hline$L A M P 2$ & $\begin{array}{l}\text { Lysosomal-associated } \\
\text { membrane protein } 2\end{array}$ & - & - & $1.79(2.2)$ & $1.63(3.7)$ & $1.56(6)$ & $2.02(3.9)$ & $3.66(2.7)$ & 6 \\
\hline LAPTM4B & $\begin{array}{l}\text { Lysosomal protein } \\
\text { transmembrane } 4 \text { beta }\end{array}$ & $1.76(7)$ & - & $1.54(3.4)$ & $1.82(2)$ & $2.31(3.3)$ & $2.07(3.8)$ & - & 0 \\
\hline CTSA & Cathepsin A & $1.98(13.6)$ & $2.13(5.3)$ & $2.26(4.8)$ & $1.81(4.3)$ & - & - & - & 4 \\
\hline GLA & galactosidase, alpha & - & $1.63(5.2)$ & $1.65(4.3)$ & $1.52(7.4)$ & $1.68(6.5)$ & - & $1.59(2.6)$ & 95 \\
\hline NEU1 & $\begin{array}{l}\text { sialidase } 1 \text { (lysosomal } \\
\text { sialidase) }\end{array}$ & - & $1.67(4.1)$ & $1.65(3.6)$ & - & $1.87(9.3)$ & - & $1.93(2.1)$ & 0 \\
\hline CTSB & cathepsin B & - & $2.41(6.2)$ & $2.05(4)$ & - & - & $1.84(5.1)$ & $1.78(3.3)$ & 2 \\
\hline ATP6AP1 & $\begin{array}{l}\text { ATPase, } \mathrm{H}+ \\
\text { transporting, lysosomal } \\
\text { accessory protein } 1\end{array}$ & - & - & $1.59(3.8)$ & - & $1.67(4.4)$ & $2.12(4.3)$ & $1.57(4.1)$ & 0 \\
\hline$L A M P 1$ & $\begin{array}{l}\text { lysosomal-associated } \\
\text { membrane protein } 1\end{array}$ & - & $1.55(2.1)$ & $1.62(3.2)$ & - & - & $1.68(5.3)$ & - & 4 \\
\hline$A P 3 B 2$ & $\begin{array}{l}\text { adaptor-related protein } \\
\text { complex } 3 \text {, beta } 2 \\
\text { subunit }\end{array}$ & - & - & $1.6(2.1)$ & $2.32(4.6)$ & $266.89(3.8)$ & - & - & 0 \\
\hline
\end{tabular}

(b) Cytoskeleton pathway

\begin{tabular}{|c|c|c|c|c|c|c|c|c|c|}
\hline $\begin{array}{l}\text { Gene } \\
\text { Symbol }\end{array}$ & Gene Name & $\# 1$ & $\# 2$ & $\# 3$ & $\# 4$ & $\# 5$ & \#6 & $\# 7$ & PubMatrix $^{*}$ \\
\hline ITGAM & $\begin{array}{l}\text { integrin, alpha } \mathrm{M} \\
\text { (complement } \\
\text { component } 3 \text { receptor } \\
3 \text { subunit) }\end{array}$ & $1.72(7.98)$ & $2.82(6.41)$ & $2.88(9.42)$ & $3.19(8.44)$ & $3.16(9.19)$ & $4.36(6.19)$ & $4.01(3.99)$ & 356 \\
\hline LIMK2 & LIM domain kinase 2 & $1.69(3.39)$ & $2.83(5.24)$ & $4.3(9.27)$ & $2.08(4.79)$ & $5.72(8.34)$ & $11.72(4.1)$ & - & 0 \\
\hline PDGFC & $\begin{array}{l}\text { platelet derived } \\
\text { growth factor C }\end{array}$ & $1.9(9.1)$ & $2.55(2.75)$ & $2.63(3.84)$ & $6.04(5.54)$ & $2.86(3.22)$ & $7.74(3.45)$ & - & 0 \\
\hline PIK3CB & $\begin{array}{l}\text { phosphatidylinositol- } \\
\text { 4,5-bisphosphate } \\
\text { 3-kinase, catalytic } \\
\text { subunit beta }\end{array}$ & - & $1.96(3.54)$ & $2.8(6.61)$ & $2.57(6.07)$ & $2.08(8.43)$ & $3.55(3.7)$ & $2.99(3.7)$ & 3 \\
\hline SOS2 & $\begin{array}{l}\text { son of sevenless } \\
\text { homolog } 2 \\
\text { (Drosophila) }\end{array}$ & $1.58(4.52)$ & $2.32(4.43)$ & $2.66(5.3)$ & $2.41(4.77)$ & $2.67(7.69)$ & $3.57(3.7)$ & - & 0 \\
\hline GSN & gelsolin & $1.87(10.35)$ & $2.09(4.28)$ & $1.63(3.04)$ & $1.59(2.47)$ & $2.09(4.99)$ & $2.93(3.74)$ & - & 2 \\
\hline SSH1 & slingshot homolog 1 & $1.67(4.24)$ & $2.17(4.97)$ & $2.12(4.54)$ & $1.68(3.17)$ & $1.69(2.17)$ & $4.85(2.4)$ & - & 0 \\
\hline FGF13 & $\begin{array}{l}\text { fibroblast growth } \\
\text { factor } 13\end{array}$ & - & $3.6(3.81)$ & $4.87(5.35)$ & $6.27(10.06)$ & $12.36(4.07)$ & $105.9(2.54)$ & - & 0 \\
\hline
\end{tabular}


(b) Continued.

\begin{tabular}{|c|c|c|c|c|c|c|c|c|c|}
\hline $\begin{array}{l}\text { Gene } \\
\text { Symbol }\end{array}$ & Gene Name & $\# 1$ & $\# 2$ & $\# 3$ & $\# 4$ & \#5 & \#6 & $\# 7$ & PubMatrix* \\
\hline DIAPH2 & $\begin{array}{l}\text { diaphanous homolog } \\
2\end{array}$ & - & $1.65(2.4)$ & $2.23(6.84)$ & $2.47(7.12)$ & $2.08(4.64)$ & $2.71(2.21)$ & - & 0 \\
\hline PAK2 & $\begin{array}{l}\text { p21 } \\
(\mathrm{CDKN} 1 \mathrm{~A}) \text {-activated } \\
\text { kinase } 2\end{array}$ & - & $1.76(4.62)$ & $1.82(5.18)$ & - & $1.88(9.03)$ & $1.70(3.78)$ & $1.84(2.27)$ & 0 \\
\hline CD14 & CD14 molecule & $1.54(5.97)$ & $2.51(4.48)$ & $2.33(3.18)$ & - & $1.54(2.56)$ & $1.77(2)$ & - & 1564 \\
\hline ITGA $2 B$ & $\begin{array}{l}\text { integrin, alpha } 2 \mathrm{~b} \\
\text { (platelet glycoprotein } \\
\text { IIb of IIb/IIIa } \\
\text { complex, antigen } \\
\text { CD41) }\end{array}$ & $3.09(9.06)$ & $1.74(2.6)$ & - & $4.42(4.9)$ & - & $3.44(3.84)$ & - & 0 \\
\hline$B R A F$ & $\begin{array}{l}\text { B-Raf } \\
\text { proto-oncogene, } \\
\text { serine/threonine } \\
\text { kinase }\end{array}$ & - & $1.94(3.7)$ & $2.2(9.68)$ & $2.68(8.92)$ & - & $1.90(2.04)$ & - & 2 \\
\hline MYL9 & $\begin{array}{l}\text { myosin, light chain } 9 \text {, } \\
\text { regulatory }\end{array}$ & $3.39(10.79)$ & - & - & $3.38(3.84)$ & $2.25(2.45)$ & $2.53(2.01)$ & - & 0 \\
\hline MAPK1 & $\begin{array}{l}\text { mitogen-activated } \\
\text { protein kinase } 1\end{array}$ & - & $2(7.85)$ & $1.85(2.74)$ & $1.79(6.61)$ & - & $1.89(4.94)$ & - & 137 \\
\hline
\end{tabular}

GEO series \#1 to 7 are listed: GSE30119, GSE8121, GSE9692, GSE28750, GSE13015-6106, GSE13015-6947 and GSE21802.

* Number of publications associated with search terms "sepsis", "severe sepsis" or "septic shock" in PubMed by searching the PubMatrix database.

analysis that considers the whole network structure to search for important nodes (hubs) that are useful as biomarkers or therapeutic targets.

We hypothesize that the changes in steady-state mRNA levels also have a profound effect on the expressed proteome. We are particularly interested in defining a set of proteins that mediate the cross-talk between lysosome and cytoskeleton pathways. Therefore, we searched for protein-protein interaction partners for the top 15 most significantly upregulated transcripts in each pathway for molecular network analysis using the "Unified Human Interactome Database" (UniHI 7) which has enhanced features allowing "Phenotype Enrichment analysis" that enables the construction of mortalityspecific networks. We identified 21 highly interconnected nodes "hubs" linking the 30 most significantly upregulated transcripts in both pathways (Figure 5 and Supplementary Figure 2). The resulting protein-protein interaction network included 10 upregulated transcripts (SORT1, CTSA, CTSB, CTSD, CD63, GNS, GLA, NEU1, ATP6AP1, and LAMP1) in the lysosome pathway and 12 upregulated transcripts (ITGAM, LIMK2, PIK3CB, SOS2, GSN, SSH1, PAK2, CD14, ITGA2B, BRAF, MYL9, and MAPK1) in the cytoskeleton pathway (Figure 5). Importantly, Phenotype Enrichment analysis revealed that a subset of the transcripts (including $M A P K 1, C T S A$, and NEU1 among the top transcripts) in this highly coordinated network was enriched for mortality.

Prior evidence was found supporting the role of MAPK signaling pathway molecule MAPK1 in regulating cytoskeletal rearrangements and sepsis mortality. The MAPK signaling pathway is believed to play an important role in mediating proinflammatory responses and is drug targets for treatments of sepsis. MAPK1 (ERK2) and MAPK3 (ERK1) are the 2 MAPKs, which play an important role in the MAPK/ERK cascade. Depending on the cellular context, the MAPK/ERK cascade mediates diverse biological functions such as cell growth, adhesion, survival, and differentiation through the regulation of transcription, translation, and cytoskeletal rearrangements. In CLP-treated septic mice, p38 MAPK inhibitor (SB203580) significantly inhibited high mobility group box 1 (HMGB-1) release and increased survival rate [41], HMGB-1 is an endogenous ligand for TLR4, in addition to LPS, and an important mediator of sepsis-associated death in experimental studies [42]. In a human model of endotoxemia, the oral administration of a new 338 MAPK inhibitor reduced cytokine production and leukocyte responses. Additionally, many of the interacting molecules in this network were drug targets. However, the most significantly upregulated transcripts identified in our study had not been directly targeted by any drug treatments (Figure 2 in Supplementary Material), suggesting more studies are warranted targeting these dysregulated molecules as novel therapeutic targets for sepsis.

\section{Discussion}

Sepsis and septic shock are a major cause of mortality in intensive care units. The pathophysiology of sepsis involves highly complex interactions between invading microorganisms, the innate and adaptive immune systems of the host, and multiple downstream events leading to organ dysfunction [43]. Despite major advances in our understanding of the pathophysiology of sepsis, we still lack the tools and indicators for early diagnosis. Gene profiling of human peripheral blood cells has been successfully employed for biomarker 

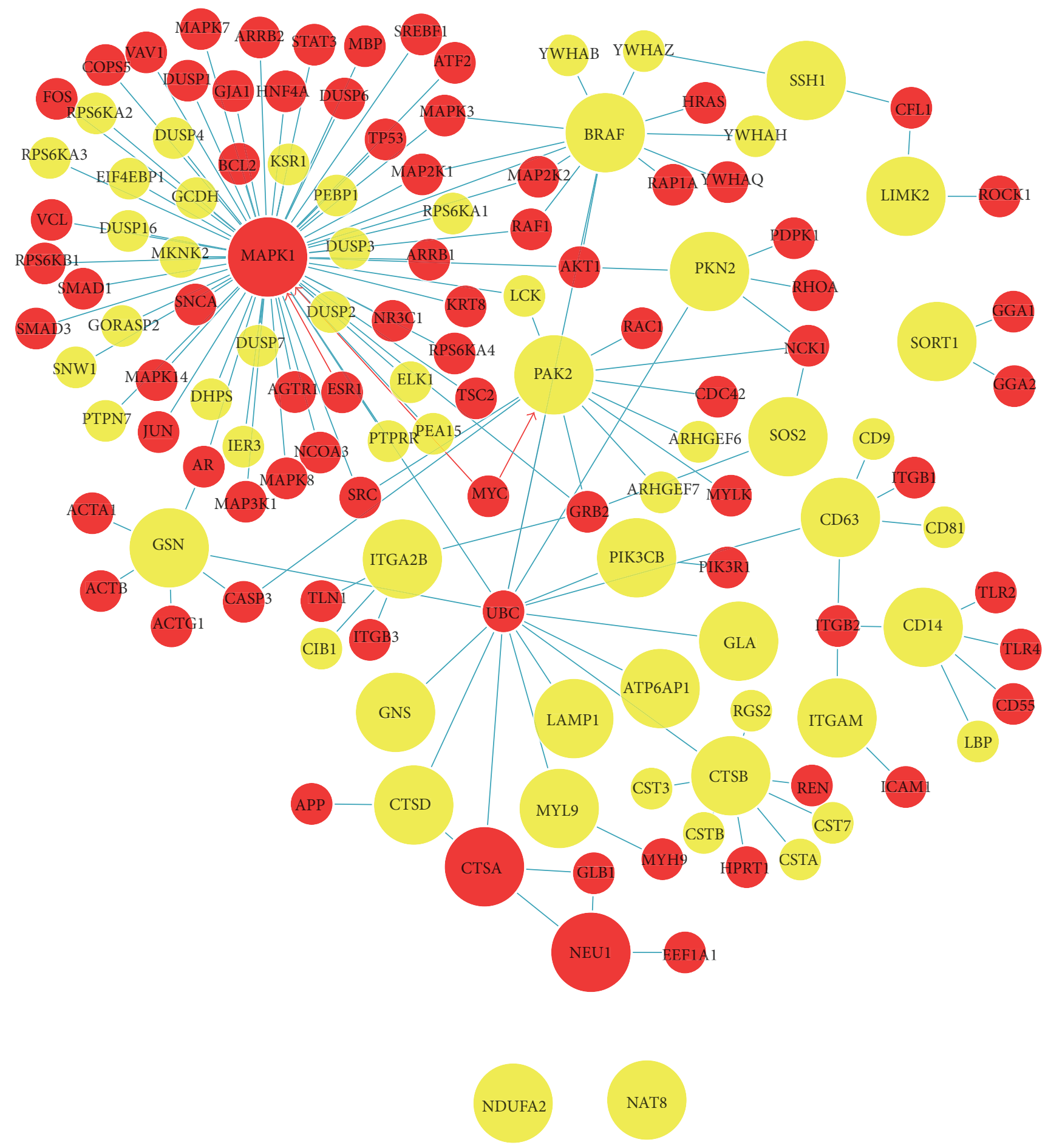

FIGURE 5: Graphical representations of protein interactions enriched for mortality phenotype. We queried the Unified Human Interactome database (UniHI 7) [21] with a list of top 15 transcripts from each of the two pathways. The display was restricted to those interactions (yellow proteins) supported by at least three PubMed references. Molecular network analysis revealed that 22 out of 30 transcripts formed a network with multiple interconnected nodes (hubs) between top genes in both pathways. Importantly, many of the predicted interaction partners (red proteins), including 3 query proteins (MAPK1, CTSA, and NEU1), were associated with the mortality phenotype. 
discovery in various lung diseases including asthma [44], pulmonary hypertension [45], and COPD [46]. To investigate whether peripheral blood can serve as an accessible surrogate tissue outside of the lung for noninvasive discovery of molecular biomarkers for sepsis, we performed a meta-analysis of gene expression microarray data from human whole blood studies of sepsis, to identify specific pathways most implicated in sepsis. Our data provides evidence that gene expression profiling using whole blood can help elucidate novel pathophysiological mechanisms that may play a critical role in sepsis.

A complex network of biological mediators underlies the clinical syndrome of sepsis. Biomarker discovery utilizing systems-level approaches promises to transform sepsis from a physiologic syndrome to a group of distinct biochemical disorders. Furthermore, greater understanding of the complex network of immune, inflammation, coagulation, intermediary metabolism and other specific mediators of sepsis may allow the development of rational and novel therapies [47, 48]. Most of the distinct molecules proposed as useful biological markers of sepsis were implicated in the immunoinflammatory process common to sepsis including the production of proinflammatory cytokines, adhesion molecules, vasoactive mediators and reactive oxygen species [49]. More systematic investigations are needed to unravel the nature of the dysregulated molecular interaction networks during sepsis.

A systematic review [11] of 12 genomic studies that examined the host response of circulating leukocytes to human sepsis showed that there was an immediate activation of pathogen recognition receptors, such as TLRs and CD14, accompanied by an increase in the activities of signal transduction cascades (included NF- $\kappa \mathrm{B}$, MAPK, JAK and STAT pathways), a process essential for subsequent transcription of immune response genes. In contrast, sepsis related inflammatory changes are highly variable on a transcriptional level. Established inflammatory markers, such as TNF- $\alpha$, IL-1 or IL-10, did not show any consistent pattern in their gene-expression across cohorts [11]. In our meta-analysis, a consistent pattern of over-expression was seen for pathways well known to be involved in sepsis (e.g., TLR and MAPK signaling) as well as for the lysosome and cytoskeleton pathways, which had not been recognized in individual studies of sepsis reported previously. Additionally, the "Ribosome," "Spliceosome," and "Cell adhesion molecules (CAMs)" pathways were downregulated across most of the datasets examined, which were also novel findings revealed through the meta-analysis. These findings suggest overall there was an imbalance between the rate of protein synthesis and protein degradation or proteolysis, which can lead to protein wasting and multiple organ system dysfunction during sepsis [50]. The manifestation in skeletal muscle is a loss of skeletal muscle mass producing diminished muscle strength [51-53]. Sepsis induces the loss of muscle proteins by impairing skeletal muscle protein synthesis through an inhibition of messenger RNA (mRNA) translation by the ribosome [54]. Muscle weakness in septic patients contributes to a continued dependence on mechanical respirators, an increased risk of pneumonia, and the complications associated with reduced ability for ambulation. These clinical complications prolong hospitalization and convalescence, thereby increasing healthcare costs [55].

Our meta-analysis included 6 studies utilizing whole blood samples. In addition to activation of pathways related to inflammation, signaling, and immunity, which were common pathways across all 6 studies examined, we found robust upregulation of the "Lysosome" and "Regulation of actin cytoskeleton" pathways in all these studies. Lysosomes are ubiquitous membrane-bound intracellular organelles containing more than 40 hydrolases in an acidic environment ( $\mathrm{pH}$ of about 5, Figure 4). They are central for degradation and recycling of macromolecules delivered by endocytosis, phagocytosis, and autophagy. Of note, both the "Phagocytosis" and "Endocytosis" pathways were upregulated in our study (Table 2), along with the "Lysosome" pathway. Further, lysosomes contribute to cellular homeostasis by way of their involvement in secretion, plasma membrane repair, cell signaling and energy metabolism, which are related to health and diseases [56]. The severe consequences of inadequate lysosome function are seen in genetically inherited lysosomal storage diseases (LSD). The primary cause is deficiency of an acidic hydrolase, while there are also conditions arising from defects in lysosomal membrane proteins that fail to transport an essential enzyme [57]. The function of lysosomes is critically dependent on soluble lysosomal hydrolases (e.g., cathepsins) as well as lysosomal membrane proteins (e.g., lysosome-associated membrane proteins) [58]. In contrast to the rather simplified view of lysosomes as simply sites for disposal of macromolecules that have been designated for degradation, lysosomes are now recognized as advanced organelles involved in many cellular processes and are considered crucial regulators of cell homeostasis. There is increasing evidence indicating that lysosomes are involved in more widespread diseases, such as cancer, Alzheimer's disease [59], and amyotrophic lateral sclerosis [60]. Moreover, due to the essential role of lysosomes in autophagy, lysosomal dysfunction impairs this process, thereby contributing to disease [61]. In our study, we found that overall lysosomal pathway was activated in human sepsis with multiple lysosomal hydrolases and membrane proteins involved (Figure 4), suggesting the implication of lysosome system dysfunction to the pathogenesis of sepsis. It has been demonstrated recently in rats that a hyper consumption and subsequent reformation of the lysosome to meet the increased demand for autophagosome cleaning, a cellular defense system to prevent harmful effects, may be involved in cardioprotection against LPS-induced septic insults [62]. Another study [63] also demonstrated that $90 \%$ of intracellular bacteria were killed within 3 hours after Human Brain Microvascular Endothelial Cells were infected with S. pneumoniae and inhibition of lysosomal function resulted in a great increase of intracellular bacteria survival suggesting that the bacteria was destroyed in the lysosome. In vivo, it was also proved that lysosome played a protective role against sepsis. The levels of autolysosome were significantly increased in the liver of septic mice induced by CLP and inhibition of lysosomal function caused higher level of aspartate transaminase and reduced mice survival [64]. 
The neutrophil is a key contributor to the innate immune response and plays an important role in the pathogenesis of sepsis-induced multiple organ dysfunction. Specific inflammatory stimuli such as LPS, cytokines, chemokines and the adhesion molecules can lead to upregulation of neutrophil activity; drive pulmonary neutrophil transendothelial migration (TEM), a paradigm of rolling/adhering/diapedesis, and neutrophil-mediated inflammation leading to multiple tissue damages, organ failures and finally to death [65]. Notably, the "Leukocyte transendothelial migration" pathway was significantly upregulated in our study (Table 2), suggesting neutrophil activation in blood of human sepsis. The antimicrobial activity of the neutrophil is nonselective, so the activity of neutrophil-mediated inflammation must be regulated to prevent tissue injury. Pathogens are degraded into fragments and these nonhost peptides processed via the endosomal pathway become antigens that, in turn, are presented to Tcells. The antigen presenting nature of neutrophils during inflammation is supported by evidence that neutrophils contain reserves of cathepsin $\mathrm{B}$ and $\mathrm{D}$, lysosomal proteases necessary for antigen presentation [66]. Cathepsins are a group of lysosomal proteases that have a key role in cellular protein turnover. The main function of cathepsins is protein recycling within the lysosome but they are also known to be involved in a range of other physiological, as well as pathological processes, including sepsis. In addition to the serine proteases cathepsins $\mathrm{A}$ and $\mathrm{G}$, and ubiquitous aspartic proteases cathepsins D and E (only present in endosomes), the most important are cysteine cathepsins. In humans, there are 11 cysteine cathepsins known at the sequence level including cathepsins B, C (dipeptidyl peptidase I, DPPI) [67]. Cathepsin C plays an important role in bacterial killing and immune regulation, the activities of three serine proteases (neutrophil elastase, cathepsin $\mathrm{G}$, and proteinase 3); all require cathepsin $\mathrm{C}$ activity for processing and maturity. It was found in mice that restricting cathepsin $C$ activity could negatively affect neutrophil bactericidal killing activity [68]. In our study, both cathepsins A and D were among the top dysregulated molecules in the lysosome pathway in human sepsis. While the pathogenesis of sepsis is increasingly well understood, the involvement of the lysosomal pathway in neutrophilmediated inflammatory diseases remains incompletely characterized, as well as its dynamic interaction with other major pathways (e.g., cytoskeleton and MAP-Kinase signaling).

During sepsis-causing infections, the vasculature is profoundly altered by the combination of microbial virulence factors and proinflammatory mediators released from activated blood cells that gain access to surrounding tissue by crossing the leaky endothelial boundary. Severe endothelial dysfunction then results from the loss of homeostatic function of the microvascular endothelium and contributes to hypoxic injury of multiple organs. The cytoskeleton is a necessarily dynamic structure for cells which plays a crucial role in regulating permeability [69] of cells under physiologic and pathophysiologic conditions. The cytoskeleton pathway is a known modulator of endothelial barrier function and microvascular permeability [70]. Recent compelling evidence suggests that the breakdown in blood/endothelial barrier function plays a crucial role in the pathogenesis of sepsis and organ dysfunction [71] and could be a new target for sepsis therapeutics [72]. It is well known that an inflammatory stimulus leads to development of capillary leak and tissue edema due to cytoskeleton rearrangement, which is a characteristic feature of sepsis and its development is one of the causes of organ failure in that syndrome [9]. Capillary leak may also result from endothelial cells injury and death [73]. Nonmuscle myosin light-chain kinase (MYLK) mediates increased lung vascular endothelial permeability in LPS-induced lung inflammatory injury and it was demonstrated that MYLK was also essential for neutrophil transmigration [74]. We have reported previously that MYLK genetic variants conferred risk for severe sepsis and sepsis-associated acute lung injury $[75,76]$. Thus, investigation of the interaction among these key regulatory pathways is challenging and could result in significant protein remodeling in the blood and could have a profound impact on inflammation, coagulation and morbidity in sepsis.

The source of variation in gene expression studies in sepsis includes heterogeneity of the causes and microbiology of sepsis; variability in time from onset of sepsis to time of blood draw; and differences between tissues and variability in sample size [77]. There are limitations in our study, which depended on the particular available datasets. In particular, sepsis-associated organ dysfunction is the main cause of high mortality and therefore has been given particular attention $[1-3,7]$. However, no dataset in GEO related to sepsisassociated organ dysfunction met our criteria for inclusion in our meta-analysis, so we were unable to examine target genes specific for severity and outcome of sepsis. The network analysis revealed complex interaction among target molecules and cross-talk between lysosome and cytoskeleton pathways. Most importantly, this highly coordinated interaction network was enriched for the mortality phenotype and drug targets and provided additional molecular targets for therapeutic interventions for sepsis. Validation of identified novel targets as potential biomarkers for an early diagnosis of sepsis utilizing standardized biomarker methodologies, and integration of biomarkers into clinical studies (in particular, early phase studies) are warranted.

\section{Conclusions}

Our meta-analysis has gained novel insights on sepsis pathogenesis and confirmed systematic changes in lysosome and cytoskeleton pathway genes in sepsis. Such insights into the molecular biology of sepsis may ultimately lead to effective antisepsis therapeutics. Our findings also suggest that blood samples may provide a means for relatively noninvasive detection of sepsis, which may aid in the development of tests providing early diagnosis and thereby better treatment outcomes in sepsis.

\section{Abbreviations}

LPS: Lipopolysaccharide

GEO: Gene Expression Omnibus

CLP: Cecal ligation and puncture 
DAVID: Database for Annotation, Visualization, and Integrated Discovery

MSI: $\quad$ Mean signal intensity

SAM: $\quad$ Significance Analysis of Microarray

FDRs: $\quad$ False discovery rates

KEGG: Kyoto Encyclopedia of Genes and Genomes

PBMCs: Peripheral blood mononuclear cells

HMVECs: Human lung microvascular endothelial cells.

\section{Conflict of Interests}

Authors declare that there is no conflict of interests regarding the publication of this paper.

\section{Authors' Contribution}

The project was conceived and planned by Li Gao, Jie Ma, and Xiangdong Guan Bioinformatics and statistical analysis was performed by Jie Ma, Chuanxi Chen, Chris Cheadle, and Andreas S. Barth. The paper was written by Jie Ma and Li Gao. All authors read and approved the final paper. Chuanxi Chen and Jie Ma equally contributed to this paper.

\section{Acknowledgments}

The authors thank Dr. Alan E. Berger for helpful reading and comments. This work was supported in part by NIH Grant no. 1R21AI110844 (to Li Gao) and Grant no. 81071536 from the National Natural Science Foundation of China (to Xiangdong Guan).

\section{References}

[1] T. Lagu, M. B. Rothberg, M. S. Shieh, P. S. Pekow, J. S. Steingrub, and P. K. Lindenauer, "Hospitalizations, costs, and outcomes of severe sepsis in the United States 2003 to 2007," Critical Care Medicine, vol. 40, no. 3, pp. 754-761, 2012.

[2] G. Kumar, N. Kumar, A. Taneja et al., "Nationwide trends of severe sepsis in the 21st century (2000-2007)," Chest, vol. 140, no. 5, pp. 1223-1231, 2011.

[3] H.-N. Shen, C.-L. Lu, and H.-H. Yang, "Epidemiologic trend of severe sepsis in Taiwan from 1997 through 2006," Chest, vol. 138, no. 2, pp. 298-304, 2010.

[4] M. Heron, D. L. Hoyert, S. L. Murphy et al., "Deaths: final data for 2006," National Vital Statistics Reports, vol. 57, no. 14, pp. 1134, 2009.

[5] E. K. Stevenson, A. R. Rubenstein, G. T. Radin, R. S. Wiener, and A. J. Walkey, "Two decades of mortality trends among patients with severe sepsis: a comparative meta-analysis," Critical Care Medicine, vol. 42, no. 3, pp. 625-631, 2014.

[6] A. Melamed and F. J. Sorvillo, "The burden of sepsis-associated mortality in the United States from 1999 to 2005: an analysis of multiple-cause-of-death data," Critical Care, vol. 13, no. 1, article R28, 2009.

[7] V. Sundararajan, C. M. MacIsaac, J. J. Presneill, J. F. Cade, and K. Visvanathan, "Epidemiology of sepsis in Victoria, Australia," Critical Care Medicine, vol. 33, no. 1, pp. 71-80, 2005.

[8] B. Cheng, G. Xie, S. Yao et al., "Epidemiology of severe sepsis in critically ill surgical patients in ten university hospitals in
China," Critical Care Medicine, vol. 35, no. 11, pp. 2538-2546, 2007.

[9] T. Gustot, "Multiple organ failure in sepsis: prognosis and role of systemic inflammatory response," Current Opinion in Critical Care, vol. 17, no. 2, pp. 153-159, 2011.

[10] M. M. Wurfel, "Genetic insights into sepsis: what have we learned and how will it help?" Current Pharmaceutical Design, vol. 14, no. 19, pp. 1900-1911, 2008.

[11] B. M. Tang, S. J. Huang, and A. S. McLean, "Genome-wide transcription profiling of human sepsis: a systematic review," Critical Care, vol. 14, no. 6, article R237, 2010.

[12] A. Castoldi, T. T. Braga, M. Correa-Costa et al., "TLR2, TLR4 and the Myd88 signaling pathway are crucial for neutrophil migration in acute kidney injury induced by sepsis," PLOS ONE, vol. 7, no. 5, Article ID e37584, 2012.

[13] D. Karmarkar and K. L. Rock, "Microbiota signalling through MyD88 is necessary for a systemic neutrophilic inflammatory response," Immunology, vol. 140, no. 4, pp. 483-492, 2013.

[14] J. Xing and A. A. Birukova, "ANP attenuates inflammatory signaling and Rho pathway of lung endothelial permeability induced by LPS and TNFalpha," Microvascular Research, vol. 79, no. 1, pp. 56-62, 2010.

[15] A. DebRoy, S. M. Vogel, D. Soni, P. C. Sundivakkam, A. B. Malik, and C. Tiruppathi, "Cooperative signaling via transcription factors NF- $\kappa$ B and AP1/c-Fos mediates endothelial cell STIM1 expression and hyperpermeability in response to endotoxin," The Journal of Biological Chemistry, vol. 289, no. 35, pp. 2418824201, 2014.

[16] N. Schlegel, R. Leweke, M. Meir, C.-T. Germer, and J. Waschke, "Role of NF- $\kappa$ B activation in LPS-induced endothelial barrier breakdown," Histochemistry and Cell Biology, vol. 138, no. 4, pp. 627-641, 2012.

[17] A. W. O'Sullivan, J. H. Wang, and H. P. Redmond, "NF- $\kappa$ B and p38 MAPK inhibition improve survival in endotoxin shock and in a cecal ligation and puncture model of sepsis in combination with antibiotic therapy," Journal of Surgical Research, vol. 152, no. 1, pp. 46-53, 2009.

[18] S. F. Liu and A. B. Malik, "NF-kappaB activation as a pathological mechanism of septic shock and inflammation," The American Journal of Physiology-Lung Cellular and Molecular Physiology, vol. 290, no. 4, pp. L622-L645, 2006.

[19] M. Schouten, W. J. Wiersinga, M. Levi, and T. van der Poll, "Inflammation, endothelium, and coagulation in sepsis," Journal of Leukocyte Biology, vol. 83, no. 3, pp. 536-545, 2008.

[20] M. Levi and T. Van Der Poll, "Inflammation and coagulation," Critical Care Medicine, vol. 38, no. 2, supplement, pp. S26-S34, 2010.

[21] R. K. R. Kalathur, J. P. Pinto, M. A. Hernández-Prieto et al., "UniHI 7: an enhanced database for retrieval and interactive analysis of human molecular interaction networks," Nucleic Acids Research, vol. 42, no. 1, pp. D408-D414, 2014.

[22] R. Edgar, M. Domrachev, and A. E. Lash, "Gene Expression Omnibus: NCBI gene expression and hybridization array data repository," Nucleic Acids Research, vol. 30, no. 1, pp. 207-210, 2002.

[23] T. P. Shanley, N. Cvijanovich, R. Lin et al., "Genome-level longitudinal expression of signaling pathways and gene networks in pediatric septic shock," Molecular Medicine, vol. 13, no. 9-10, pp. 495-508, 2007.

[24] N. Cvijanovich, T. P. Shanley, R. Lin et al., "Validating the genomic signature of pediatric septic shock," Physiological Genomics, vol. 34, no. 1, pp. 127-134, 2008. 
[25] A. Sutherland, M. Thomas, R. A. Brandon et al., "Development and validation of a novel molecular biomarker diagnostic test for the early detection of sepsis," Critical Care, vol. 15, no. 3, article R149, 2011.

[26] R. Pankla, S. Buddhisa, M. Berry et al., "Genomic transcriptional profiling identifies a candidate blood biomarker signature for the diagnosis of septicemic melioidosis," Genome Biology, vol. 10, no. 11, article R127, 2009.

[27] J. F. Bermejo-Martin, I. Martin-Loeches, J. Rello et al., "Host adaptive immunity deficiency in severe pandemic influenza," Critical Care, vol. 14, no. 5, article R167, 2010.

[28] R. Banchereau, A. Jordan-Villegas, M. Ardura et al., "Host immune transcriptional profiles reflect the variability in clinical disease manifestations in patients with staphylococcus aureus infections," PLoS ONE, vol. 7, no. 4, Article ID e34390, 2012.

[29] V. G. Tusher, R. Tibshirani, and G. Chu, "Significance analysis of microarrays applied to the ionizing radiation response," Proceedings of the National Academy of Sciences of the United States of America, vol. 98, no. 9, pp. 5116-5121, 2001.

[30] A. S. Barth, A. Kumordzie, C. Colantuoni, K. B. Margulies, T. P. Cappola, and G. F. Tomaselli, "Reciprocal regulation of metabolic and signaling pathways," BMC Genomics, vol. 11, no. 1, article 197, 2010.

[31] M. Kanehisa and S. Goto, "KEGG: kyoto encyclopedia of genes and genomes," Nucleic Acids Research, vol. 28, no. 1, pp. 27-30, 2000.

[32] G. Dennis Jr., B. T. Sherman, D. A. Hosack et al., "DAVID: database for annotation, visualization, and integrated discovery," Genome Biology, vol. 4, no. 5, p. P3, 2003.

[33] A. S. Barth, A. Kumordzie, C. Frangakis, K. B. Margulies, T. P. Cappola, and G. F. Tomaselli, "Reciprocal transcriptional regulation of metabolic and signaling pathways correlates with disease severity in heart failure," Circulation: Cardiovascular Genetics, vol. 4, no. 5, pp. 475-483, 2011.

[34] A. Sturn, J. Quackenbush, and Z. Trajanoski, "Genesis: cluster analysis of microarray data," Bioinformatics, vol. 18, no. 1, pp. 207-208, 2002.

[35] F. Ishikawa and S. Miyazaki, "New biodefense strategies by neutrophils," Archivum Immunologiae et Therapia Experimentalis (Warszawa), vol. 53, no. 3, pp. 226-233, 2005.

[36] M. Mussap, A. Noto, M. Fravega, and V. Fanos, "Soluble CD14 subtype presepsin (sCD14-ST) and lipopolysaccharide binding protein (LBP) in neonatal sepsis: new clinical and analytical perspectives for two old biomarkers," The Journal of MaternalFetal \& Neonatal Medicine, vol. 24, supplement 2, pp. 12-14, 2011.

[37] L. G. D. G. Romualdo, P. E. Torrella, M. V. González et al., "Diagnostic accuracy of presepsin (soluble CD14 subtype) for prediction of bacteremia in patients with systemic inflammatory response syndrome in the Emergency Department," Clinical Biochemistry, vol. 47, no. 7-8, pp. 505-508, 2014.

[38] A. C. Muller Kobold, G. Mesander, C. A. Stegeman, C. G. M. Kallenberg, and J. W. Cohen Tervaert, "Are circulating neutrophils intravascularly activated in patients with antineutrophil cytoplasmic antibody (ANCA)-associated vasculitides?" Clinical and Experimental Immunology, vol. 114, no. 3, pp. 491-499, 1998.

[39] A. C. Muller Kobold, J. E. Tulleken, J. G. Zijlstra et al., "Leukocyte activation in sepsis: correlations with disease state and mortality," Intensive Care Medicine, vol. 26, no. 7, pp. 883-892, 2000 .
[40] M. Vidal, M. E. Cusick, and A. L. Barabási, "Interactome networks and human disease," Cell, vol. 144, no. 6, pp. 986-998, 2011.

[41] Y. M. Ha, S. A. Ham, Y. M. Kim et al., “ $\beta 1$-Adrenergic receptormediated HO-1 induction, via PI3K and p38 MAPK, by isoproterenol in RAW 264.7 cells leads to inhibition of HMGB1 release in LPS-activated RAW 264.7 cells and increases in survival rate of CLP-induced septic mice," Biochemical Pharmacology, vol. 82, no. 7, pp. 769-777, 2011.

[42] J. S. Park, D. Svetkauskaite, Q. He et al., "Involvement of toll-like receptors 2 and 4 in cellular activation by high mobility group box 1 protein," The Journal of Biological Chemistry, vol. 279, no. 9, pp. 7370-7377, 2004.

[43] R. S. Hotchkiss and I. E. Karl, "The pathophysiology and treatment of sepsis," The New England Journal of Medicine, vol. 348, no. 2, pp. 138-150, 2003.

[44] S. W. Shin, T. J. Oh, S.-M. Park et al., "Asthma-predictive genetic markers in gene expression profiling of peripheral blood mononuclear cells," Allergy, Asthma and Immunology Research, vol. 3, no. 4, pp. 265-272, 2011.

[45] C. Cheadle, A. E. Berger, S. C. Mathai et al., "Erythroidspecific transcriptional changes in PBMCs from pulmonary hypertension patients," PLOS ONE, vol. 7, no. 4, Article ID e34951, 2012.

[46] S. Bhattacharya, S. Tyagi, S. Srisuma et al., "Peripheral blood gene expression profiles in COPD subjects," Journal of Clinical Bioinformatics, vol. 1, no. 1, article 12, 2011.

[47] S. E. Calvano, W. Xiao, D. R. Richards et al., "A network-based analysis of systemic inflammation in humans," Nature, vol. 437, no. 7061, pp. 1032-1037, 2005.

[48] D. Rittirsch, M. A. Flierl, and P. A. Ward, "Harmful molecular mechanisms in sepsis," Nature Reviews Immunology, vol. 8, no. 10, pp. 776-787, 2008.

[49] J. C. Marshall, J. L. Vincent, M. P. Fink et al., "Measures, markers, and mediators: Toward a staging system for clinical sepsis. A Report of the Fifth Toronto Sepsis Roundtable, Toronto, Ontario, Canada, October 25-26, 2000," Critical Care Medicine, vol. 31, no. 5, pp. 1560-1567, 2003.

[50] R. N. Cooney, S. R. Kimball, and T. C. Vary, "Regulation of skeletal muscle protein turnover during sepsis: mechanisms and mediators," Shock, vol. 7, no. 1, pp. 1-16, 1997.

[51] J. A. Windsor and G. L. Hill, "Grip strength: a measure of the proportion of protein loss in surgical patients," British Journal of Surgery, vol. 75, no. 9, pp. 880-882, 1988.

[52] W. Brough, G. Horne, A. Blount, M. H. Irving, and K. N. Jeejeebhoy, "Effects of nutrient intake, surgery, sepsis, and long term administration of steroids on muscle function," British Medical Journal, vol. 293, no. 6553, pp. 983-988, 1986.

[53] M. Klaude, M. Mori, I. Tjader, T. Gustafsson, J. Wernerman, and O. Rooyackers, "Protein metabolism and gene expression in skeletal muscle of critically ill patients with sepsis," Clinical Science, vol. 122, no. 3, pp. 133-142, 2012.

[54] T. C. Vary, "Acute oral leucine administration stimulates protein synthesis during chronic sepsis through enhanced association of eukaryotic initiation factor $4 \mathrm{G}$ with eukaryotic initiation factor 4E in rats," Journal of Nutrition, vol. 137, no. 9, pp. 20742079, 2007.

[55] D. W. Wilmore, "Metabolic response to severe surgical illness: overview," World Journal of Surgery, vol. 24, no. 6, pp. 705-711, 2000 . 
[56] C. Settembre, A. Fraldi, D. L. Medina, and A. Ballabio, "Signals from the lysosome: a control centre for cellular clearance and energy metabolism," Nature Reviews Molecular Cell Biology, vol. 14, no. 5, pp. 283-296, 2013.

[57] F. M. Platt, B. Boland, and A. C. van der Spoel, "The cell biology of disease: lysosomal storage disorders: the cellular impact of lysosomal dysfunction," The Journal of Cell Biology, vol. 199, no. 5, pp. 723-734, 2012.

[58] H. Appelqvist, P. Wäster, K. Kågedal, and K. Öllinger, "The lysosome: from waste bag to potential therapeutic target," Journal of Molecular Cell Biology, vol. 5, no. 4, pp. 214-226, 2013.

[59] C. M. Bellettato and M. Scarpa, "Pathophysiology of neuropathic lysosomal storage disorders," Journal of Inherited Metabolic Disease, vol. 33, no. 4, pp. 347-362, 2010.

[60] A. Otomo, L. Pan, and S. Hadano, "Dysregulation of the autophagy-endolysosomal system in amyotrophic lateral sclerosis and related motor neuron diseases," Neurology Research International, vol. 2012, Article ID 498428, 12 pages, 2012.

[61] B. Levine and G. Kroemer, "Autophagy in the pathogenesis of disease," Cell, vol. 132, no. 1, pp. 27-42, 2008.

[62] K. Unuma, T. Aki, T. Funakoshi, K.-I. Yoshida, and K. Uemura, "Cobalt protoporphyrin accelerates TFEB activation and lysosome reformation during LPS-induced septic insults in the rat heart," PLoS ONE, vol. 8, no. 2, Article ID e56526, 2013.

[63] H. Gradstedt, F. Iovino, and J. J. E. Bijlsma, "Streptococcus pneumoniae invades endothelial host cells via multiple pathways and is killed in a lysosome dependent manner," PLOS ONE, vol. 8, no. 6, Article ID e65626, 2013.

[64] W. Takahashi, E. Watanabe, L. Fujimura et al., "Kinetics and protective role of autophagy in a mouse cecal ligation and puncture-induced sepsis," Critical Care, vol. 17, no. 4, article R160, 2013.

[65] J. G. Wagner and R. A. Roth, "Neutrophil migration mechanisms, with an emphasis on the pulmonary vasculature," Pharmacological Reviews, vol. 52, no. 3, pp. 349-374, 2000.

[66] Y. Kimura and K. Yokoi-Hayashi, "Polymorphonuclear leukocyte lysosomal proteases, cathepsins B and D affect the fibrinolytic system in human umbilical vein endothelial cells," Biochimica et Biophysica Acta-Molecular Cell Research, vol. 1310, no. 1, pp. 1-4, 1996.

[67] U. Repnik, V. Stoka, V. Turk, and B. Turk, "Lysosomes and lysosomal cathepsins in cell death," Biochimica et Biophysica Acta-Proteins and Proteomics, vol. 1824, no. 1, pp. 22-33, 2012.

[68] W. Liu, M. Yan, Y. Liu, K. R. McLeish, W. G. Coleman Jr., and G. P. Rodgers, "Olfactomedin 4 inhibits cathepsin C-mediated protease activities, thereby modulating neutrophil killing of Staphylococcus aureus and Escherichia coli in mice," Journal of Immunology, vol. 189, no. 5, pp. 2460-2467, 2012.

[69] L. S. Rodgers and A. S. Fanning, "Regulation of epithelial permeability by the actin cytoskeleton," Cytoskeleton (Hoboken), vol. 68, no. 12, pp. 653-660, 2011.

[70] J. R. Jacobson and J. G. N. Garcia, "Novel therapies for microvascular permeability in sepsis," Current Drug Targets, vol. 8, no. 4, pp. 509-514, 2007.

[71] E. de Montmollin and D. Annane, "Year in review 2010: critical care-multiple organ dysfunction and sepsis," Critical Care, vol. 15 , no. 6 , article $236,2011$.

[72] N. M. Goldenberg, B. E. Steinberg, A. S. Slutsky, and W. L. Lee, "Broken barriers: a new take on sepsis pathogenesis," Science Translational Medicine, vol. 3, no. 88, Article ID 88ps25, 2011.
[73] I. Joris, H. F. Cuenoud, G. V. Doern, J. M. Underwood, and G. Majno, "Capillary leakage in inflammation. A study by vascular labeling," American Journal of Pathology, vol. 137, no. 6, pp. 1353$1363,1990$.

[74] J. Xu, X.-P. Gao, R. Ramchandran, Y.-Y. Zhao, S. M. Vogel, and A. B. Malik, "Nonmuscle myosin light-chain kinase mediates neutrophil transmigration in sepsis-induced lung inflammation by activating $\beta 2$ integrins," Nature Immunology, vol. 9, no. 8, pp. 880-886, 2008.

[75] L. Gao, A. V. Grant, N. Rafaels et al., "Polymorphisms in the myosin light chain kinase gene that confer risk of severe sepsis are associated with a lower risk of asthma," Journal of Allergy and Clinical Immunology, vol. 119, no. 5, pp. 1111-1118, 2007.

[76] L. Gao, Y. J. Tsai, D. N. Grigoryev, and K. C. Barnes, "Host defense genes in asthma and sepsis and the role of the environment," Current Opinion in Allergy and Clinical Immunology, vol. 7, no. 6, pp. 459-467, 2007.

[77] J. A. Russell, "Gene expression in human sepsis: what have we learned?" Critical Care, vol. 15, no. 1, p. 121, 2011. 


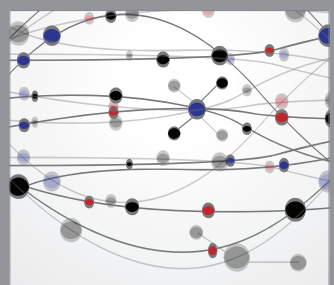

The Scientific World Journal
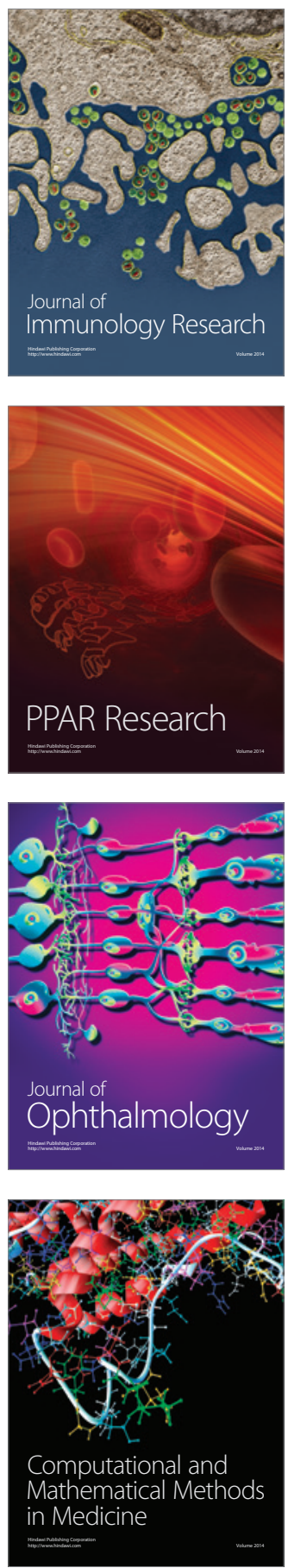

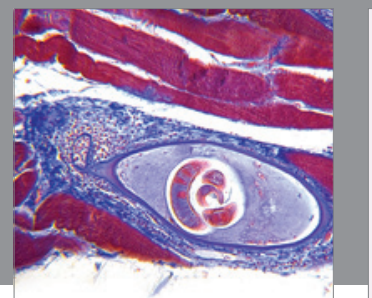

Gastroenterology

Research and Practice
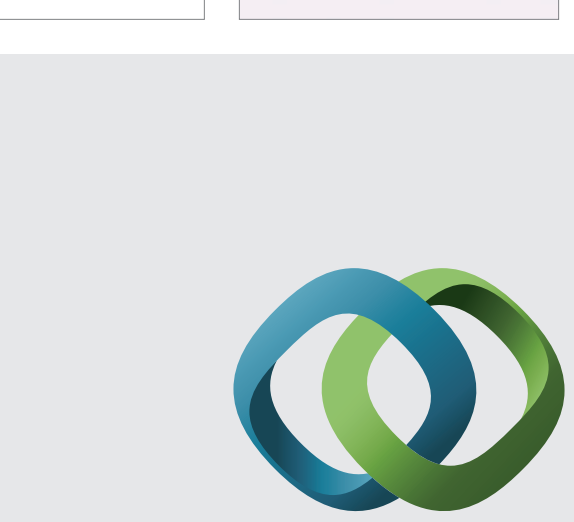

\section{Hindawi}

Submit your manuscripts at

http://www.hindawi.com
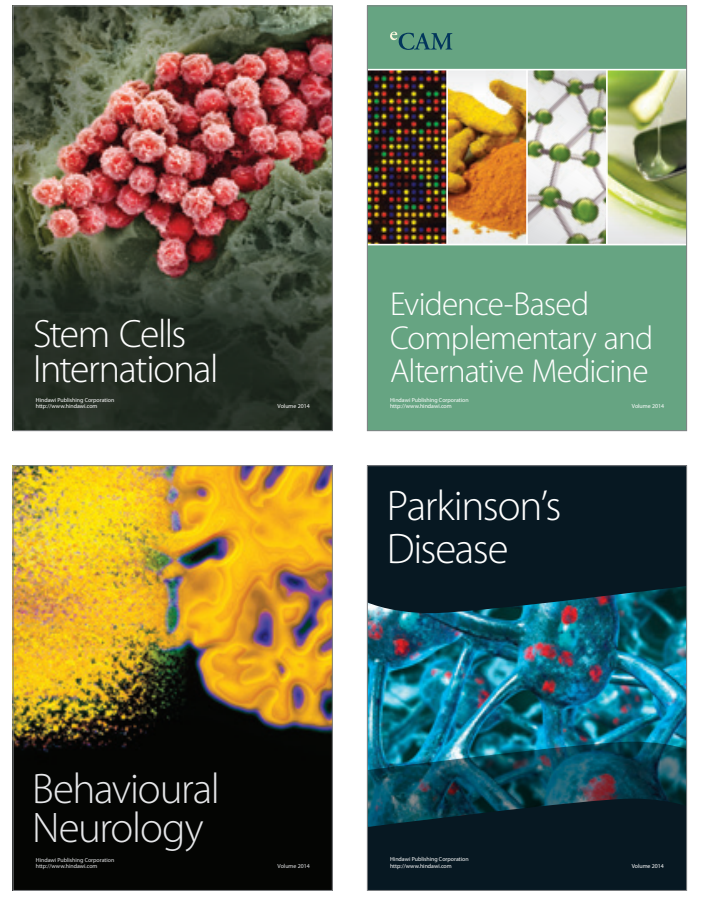
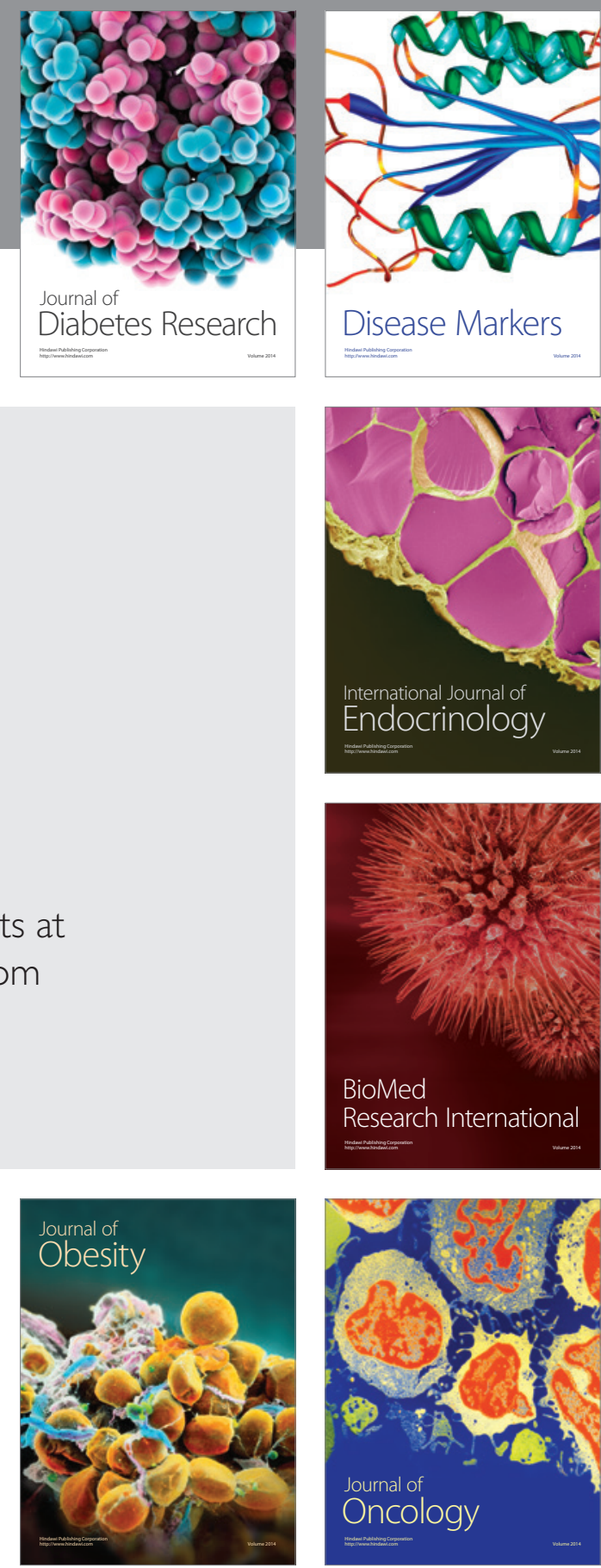

Disease Markers
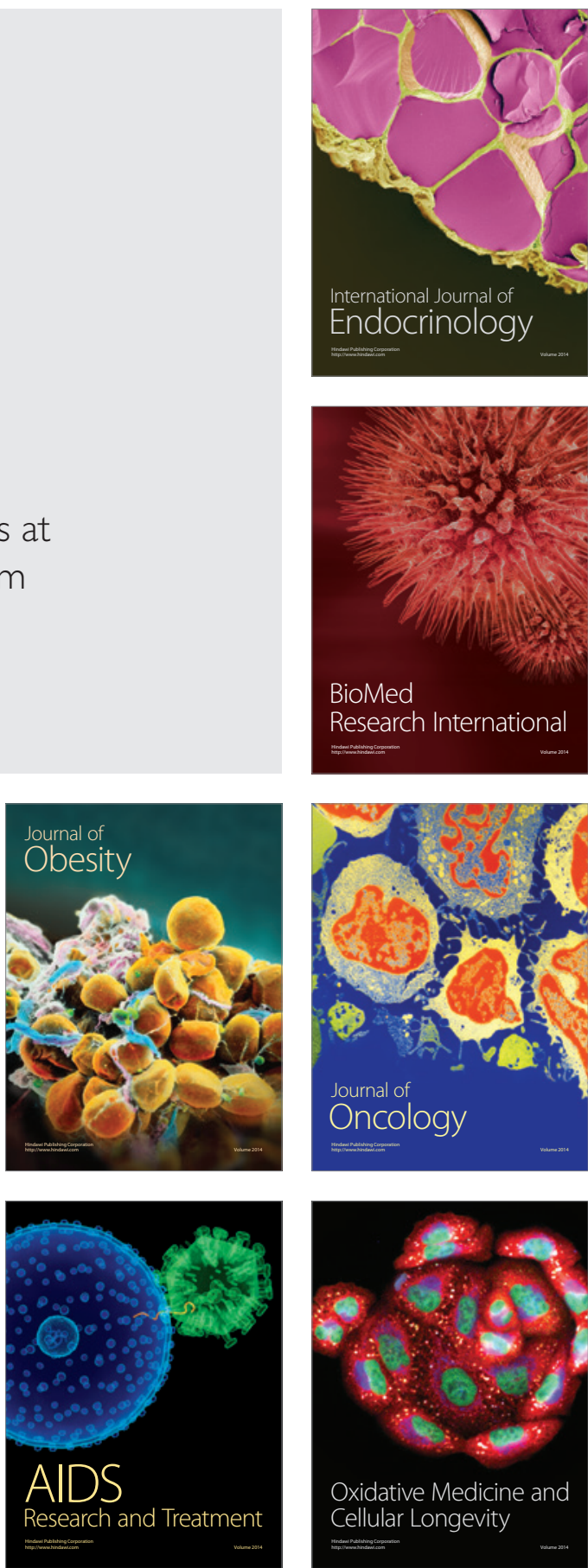